\title{
Sensitivity of the surface energy budget to drifting snow as simulated by MAR in coastal Adelie Land, Antarctica
}

Louis Le Toumelin ${ }^{1,2}$, Charles Amory ${ }^{1,3}$, Vincent Favier ${ }^{1}$, Christoph Kittel ${ }^{3}$, Stefan Hofer $^{4}$, Xavier Fettweis ${ }^{3}$, Hubert Gallée ${ }^{1}$, and Vinay Kayetha ${ }^{5}$

${ }^{1}$ Université Grenoble Alpes, CNRS, Institut des Géosciences de l'Environnement, 38000, Grenoble, France

${ }^{2}$ Univ. Grenoble Alpes, Université de Toulouse, Météo-France, CNRS, CNRM, Centre d'Études de la Neige, Grenoble, France

${ }^{3}$ F.R.S.-FNRS, Laboratory of Climatology, Department of Geography, University of Liège, 4000 Liège, Belgium ${ }^{4}$ Department of Geosciences, University of Oslo, Oslo, Norway

${ }^{5}$ Science Systems and Applications, Greenbelt, MD, USA

Correspondence: Louis Le Toumelin (louis.letoumelin@gmail.com) 


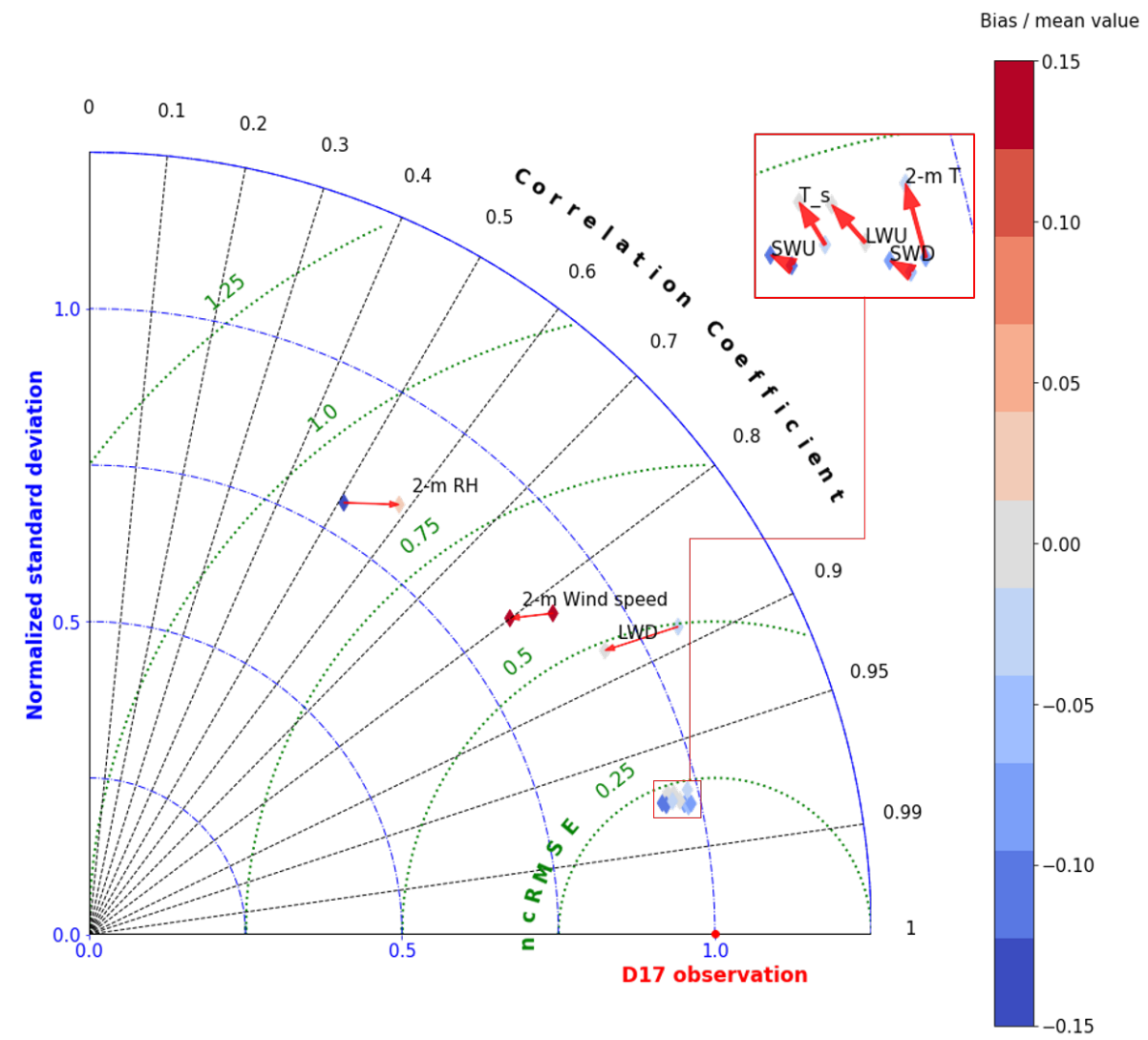

Figure S1. Taylor diagram at D17 allows visualization of modifications between simulation, using observations as a reference. The radial distance from the origin accounts for the normalized standard deviation (standard deviation of the simulated variable divided by the observed standard deviation). Correlation coefficient is represented by the angular distance from the horizontal. Normalized and centered root mean squared error (ncRMSE) is represented by a green circle centered on the red point. A simulation matching perfectly observations would stand on the red point. The colorbar indicates the mean bias divided by the mean value of observations. The arrows point from MAR-nDR simulations to MAR-DR simulations. $2 \mathrm{~m}$ RH designates $2 \mathrm{~m}$ relative humidity, $2 \mathrm{~m}$ T designates $2 \mathrm{~m}$ temperature and $T_{s}$ designates surface temperature. Surface temperature is computed using incoming and outgoing surface longwave radiations. 

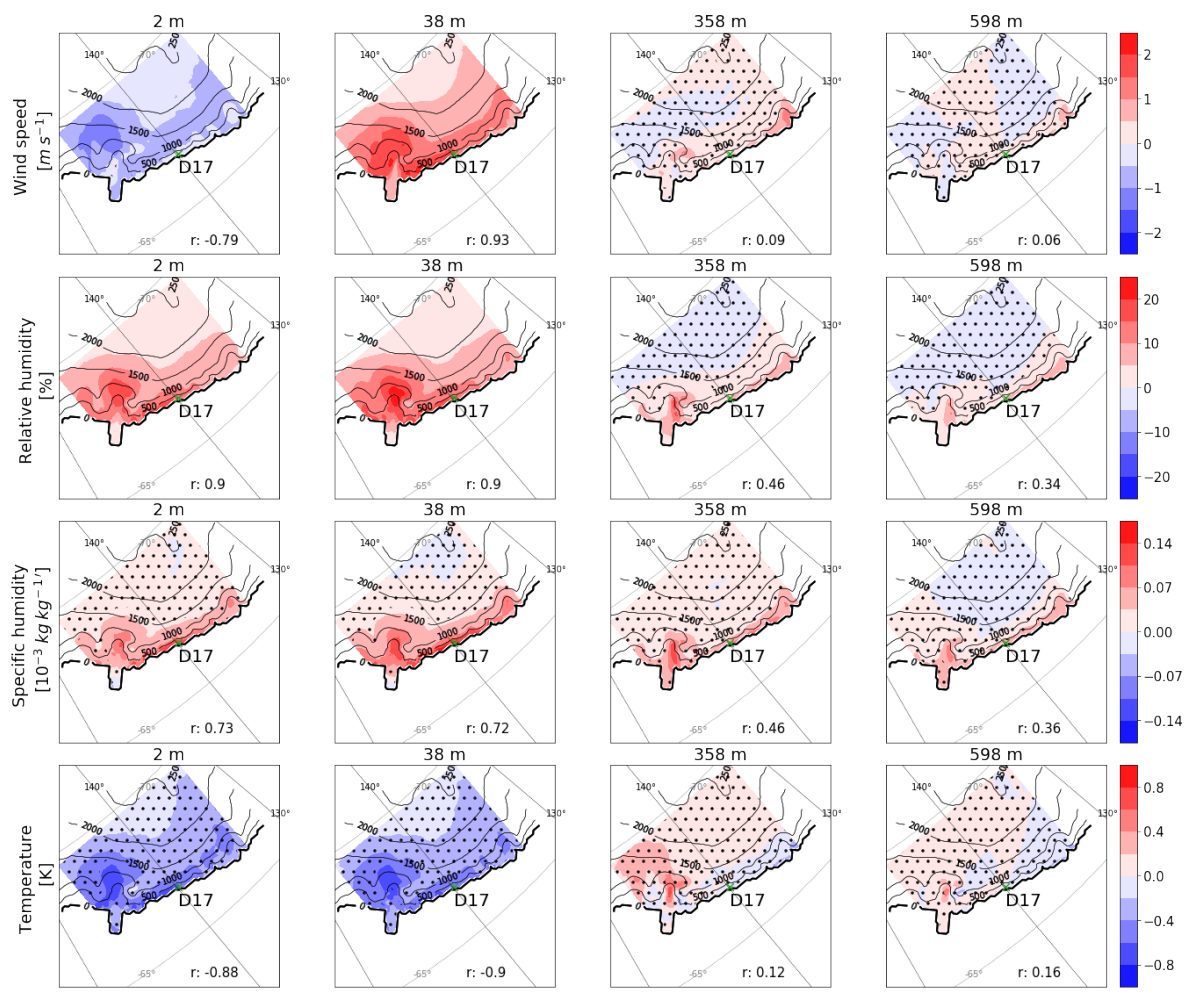

Figure S2. Modifications between MAR-DR and MAR-nDR on an integrative domain covering coastal Adelie Land computed at different vertical levels in the low-atmosphere. Within each panel, $r$ indicates the Pearson correlation coefficient between snow mass transport and the considered variable. Dotted area designate area where modifications are lower than interannual variability (taken as the standard deviation computed from annual means). 


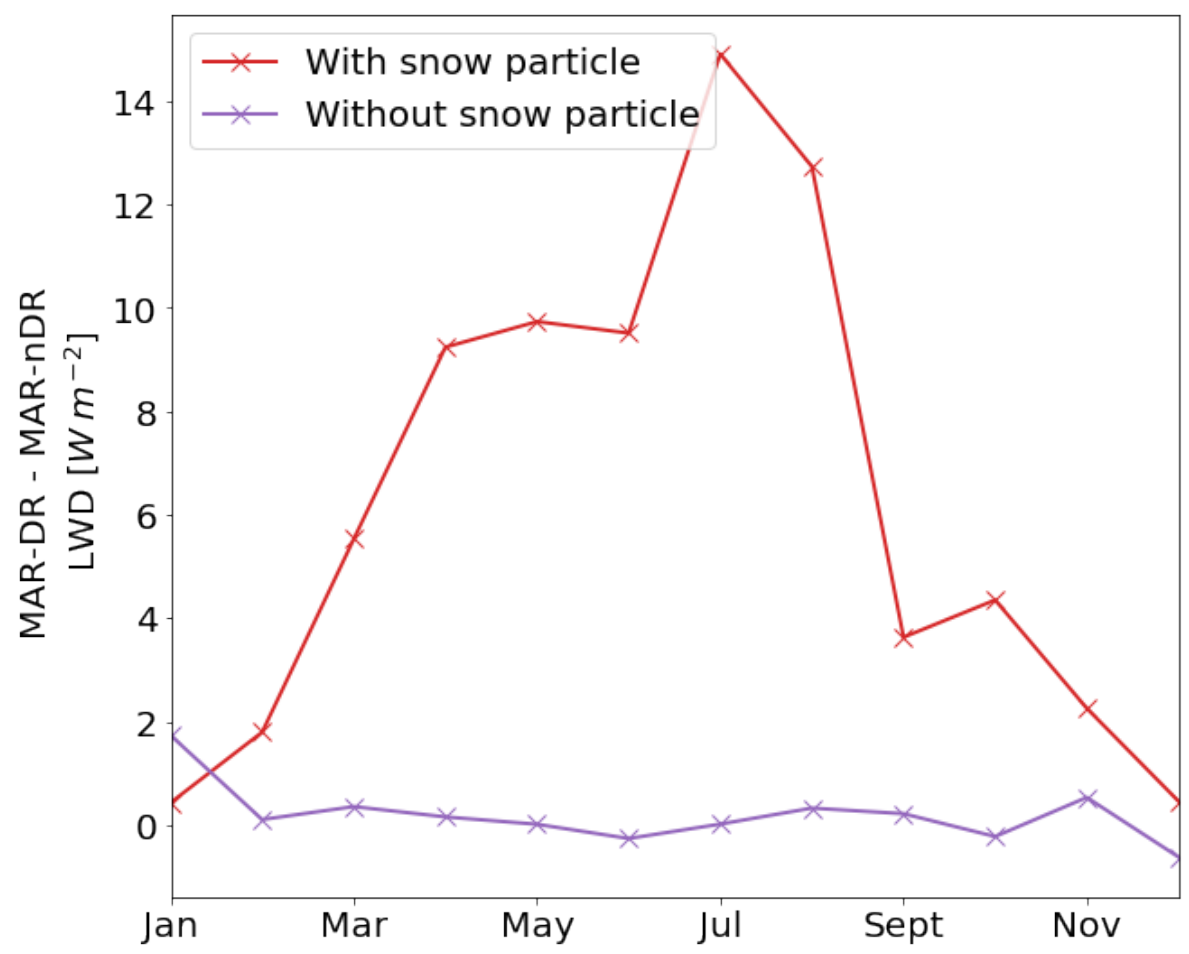

Figure S3. Time series of LWD modifications between MAR-DR and MAR-nDR at D17 for year 2017. Two model configurations are compared here: the reference simulation, referred as "With snow particle" includes the snow particle ratio in the LWD computation, oppositely to the "Without snow particle" configuration. LWD modifications between MAR-DR and MAR-nDR are highly influenced by the presence of snow particles in the atmosphere. 


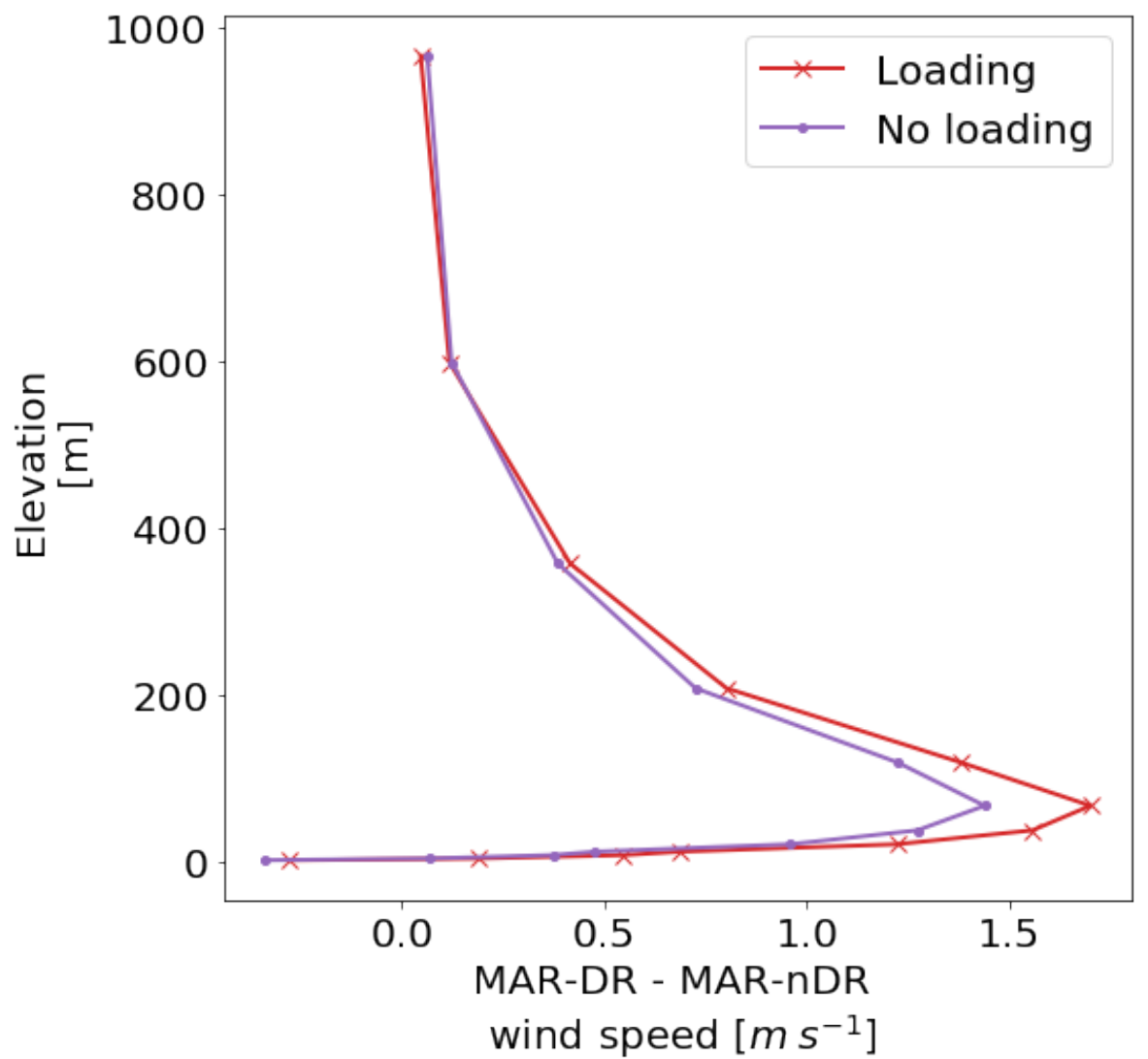

Figure S4. Vertical profile of wind speed modifications between MAR-DR and MAR-nDR at D17 for year 2017. Two model configurations are compared here: the reference simulation, referred as "loading" includes the contribution of snow particles to air density, oppositely to the "No loading" configuration. The mass of snow particle is only responsible for limited wind speed modifications when the drifting-snow scheme is activated in MAR. 


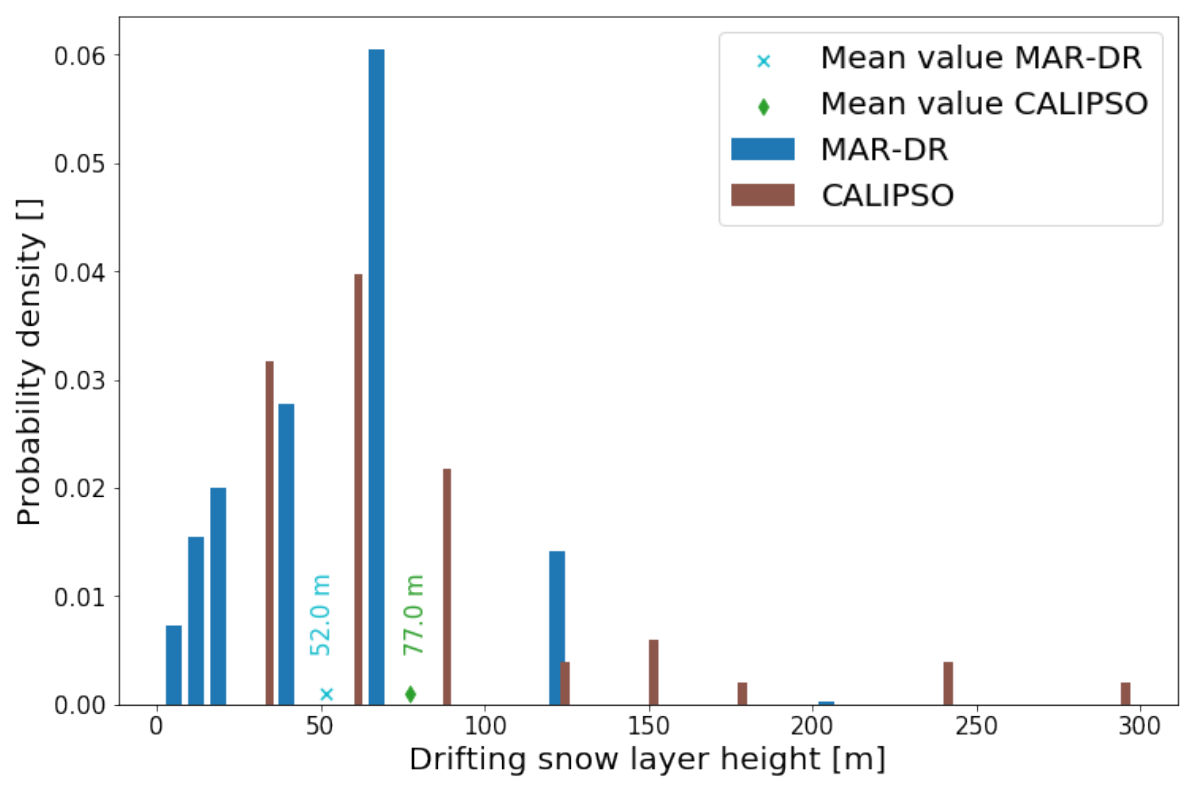

Figure S5. Distribution of drifting-snow layer heights as simulated by MAR-DR (after the filtering process described in Sect. 2.4) and as observed by CALIPSO (Palm et al., 2011). MAR-DR algorithm for detecting drifting-snow layer height is calibrated on CALIPSO observations. On the 2010-2018 period and after the filtering process, MAR-DR simulates a mean drifting-snow layer height of $52 \mathrm{~m}$ while CALIPSO detects for specifics occurrences a mean value of $77 \mathrm{~m}$. 
(a)

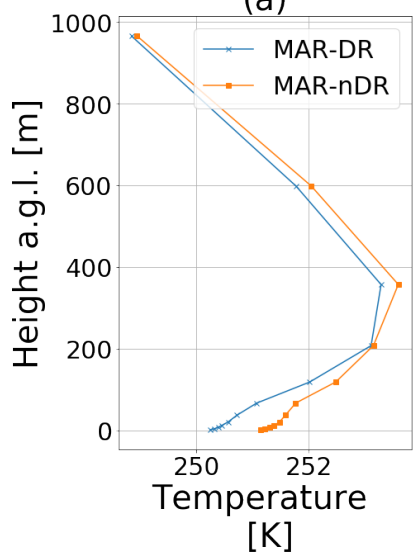

(b)

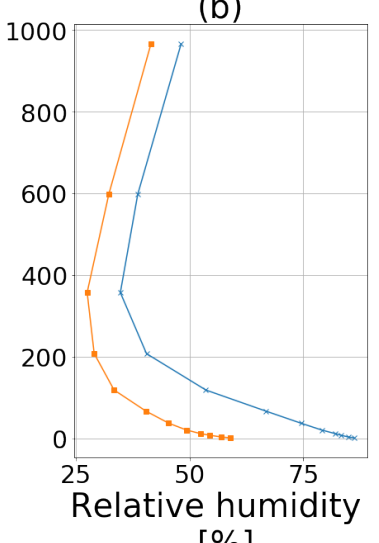

(c)

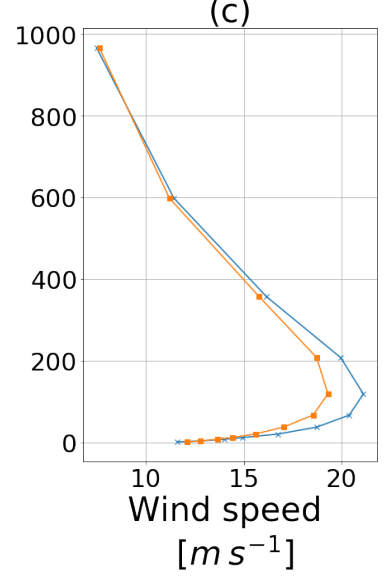

Figure S6. (a) Temperature, (b) relative humidity and (c) wind speed mean profiles calculated during a drifting-snow event between the 1st and the 3rd of October 2017 at D17. Temperature variations are small in the katabatic layer (0.01 $\mathrm{Km}^{-1}$ or less on the first $70 \mathrm{~m}$ a.g.l.), relative humidity peaks near the surface, and wind speed increases with elevation in the katabatic layer. 


\section{S0.1 Metrics}

We define ncRMSE the normal and centered root mean square error as follows:

$\mathrm{ncRMSE}=\frac{1}{s t d_{o b s}} \times\left[\frac{1}{N} \sum_{i=1}^{n}\left(\left(m_{i}-\bar{m}\right)-\left(o_{i}-\bar{o}\right)\right)^{2}\right]^{1 / 2}$

with $\mathrm{n}$ the total number of observations, $m$ the simulated value, $o$ the observed value, $\bar{m}$ the simulated mean value, $\bar{o}$ the 5 observed mean value and $s t d_{o b s}$ the observed standard deviation. 


\section{References}

Palm, S. P., Yang, Y., Spinhirne, J. D., and Marshak, A.: Satellite remote sensing of blowing snow properties over Antarctica, Journal of Geophysical Research: Atmospheres, 116, https://doi.org/10.1029/2011JD015828, 2011. 